Chronic Obstructive Pulmonary Diseases: Journal of the COPD Foundation

\author{
Original Research
}

\title{
Efficacy and Safety of Twice-Daily Glycopyrrolate Versus Placebo in Patients With COPD: The GEM2 Study
}

\author{
Edward Kerwin, $\mathrm{MD}^{1}$ Thomas M. Siler, $\mathrm{MD}^{2}$ Phillip Korenblat, $\mathrm{MD}^{3}$ Alexander White, MD ${ }^{4}$ \\ Joerg H. Eckert, $\mathrm{PhD}^{5}$ Michelle Henley, $\mathrm{MSc}^{6}$ Francesco Patalano, MD ${ }^{5}$ Peter D'Andrea, MD ${ }^{6}$
}

\section{Abstract}

Long-acting bronchodilators including muscarinic antagonists are central to the management of patients with COPD. The Glycopyrrolate Effect on syMptoms and lung function (GEM2) study assessed the efficacy and safety of twice-daily glycopyrrolate $15.6 \mu \mathrm{g}$ in patients with moderate-to-severe airflow limitation.

This 12-week multicenter, double-blind study randomized (1:1) patients to glycopyrrolate $15.6 \mu g$ twice daily (b.i.d.) or placebo both delivered via the Neohaler ${ }^{\mathrm{TM}}$ device. The primary objective was superiority of glycopyrrolate compared with placebo for forced expiratory volume in 1 second (FEV1) standardized area under curve (AUC) between 0 and 12 hours post dosing ( $\mathrm{FEV}_{1} \mathrm{AUC}_{0-12 \mathrm{~h}}$ ) at week 12. Other outcomes included additional spirometry parameters, health status using St George's Respiratory Questionnaire (SGRQ), dyspnea via Transition Dyspnea Index (TDI), rescue medication use and COPD symptoms reported by patients via the electronic diary. Safety was also assessed.

Of the 432 patients randomized (glycopyrrolate, $n=216$; placebo, $n=216$ ), $96 \%$ completed the planned treatment phase. The study met its primary objective (superiority of glycopyrrolate compared with placebo for FEV $A_{1} \mathrm{AUC}_{0-12 \mathrm{~h}}$ ). Compared with placebo, glycopyrrolate showed significant improvements in lung function parameters $(p<0.001)$. Health status (SGRQ total score and COPD assessment test), rescue medication use and daily total COPD symptom scores were significantly improved with glycopyrrolate versus placebo over 12 weeks. Improvements in dyspnea were observed with glycopyrrolate and placebo although the treatment difference was not statistically significant. Overall, differences in the incidences of adverse events and serious adverse events between the groups were not considered clinically meaningful. No deaths were reported.

Twice-daily glycopyrrolate $15.6 \mu \mathrm{g}$ showed statistically significant and clinically meaningful improvements compared with placebo in lung function, COPD symptoms, health status, and rescue medication usage in COPD patients with moderate-to-severe airflow limitation.

Clinical Trial Registration: NCT01715298

\footnotetext{
Abbreviations: Glycopyrrolate Effect on syMptoms and lung function study, GEM2; twice daily, b.i.d.; forced expiratory volume in 1 second, $\mathbf{F E V}_{\mathbf{1}}$; area under the curve, AUC; St. George's Respiratory Questionaire, SGRQ; Transition Dyspnea Index, TDI; Global initiative for chronic Lung Disease, GOLD; long-acting muscarinic antagonist, LAMA; long-acting $\beta_{2}$-agonist, LABA; inhaled corticosteroid, ICS; chronic obstructive pulmonary disease, COPD; U.S. Food and Drug Administration, FDA; forced vital capacity, FVC; modified Medical Research Council, mMRC; electronic diary, e-diary; minimal clinically important difference, MCID; COPD Assessment Test, CAT; adverse event, AE; major adverse cardiovascular events, MACE; cardio-and cerebrovascular, CCV; full analysis set, FAS; per protocol set, PPS; Baseline Dyspnea Index, BDI; glycopyrrolate, GLY; placebo, PBO; confidence interval, CI; odds ratio, OR; serious adverse effects, SAEs; Friederica's corrected QT interval, QTcF; least squares means, LSM

Funding Support: The study was supported and funded by Novartis Pharma AG, Basel. Writing support was funded by Novartis Pharma AG, Basel, Switzerland.

Date of Acceptance: December 9, 2015

Citation: Kerwin E, Siler TM, Korenblat P, et al. Efficacy and safety of twice-daily glycopyrrolate versus placebo in patients with COPD: the GEM2 study. Chronic Obstr Pulm Dis (Miami). 2016; 3(2):549-559. doi: http://dx.doi.org/10.15326/jcopdf/3.2.2015.0157
} 


\section{This article has an online data supplement.}

1 Clinical Research Institute of Southern Oregon, Medford

2 Midwest Chest Consultants, St. Charles, Missouri

3 The Clinical Research Center, St. Louis, Missouri

4 Progressive Medical Research, Port Orange, Florida

5 Novartis Pharma AG, Basel, Switzerland

6 Novartis Pharmaceuticals Corporation, East Hanover, New Jersey

\section{Address correspondence to:}

Edward Kerwin, MD

Clinical Research Institute of Southern Oregon

3860 Crater Lake Avenue

Medford, OR 97504

Phone: 541-858-1003

Email: ekerwin@criresearch.com

\section{Keywords:}

glycopyrronium; glycopyrrolate; lung function; chronic

obstructive pulmonary disease; long-acting muscarinic antagonist; bronchodilator; twice daily

\section{Introduction}

The current Global initiative for chronic Obstructive Lung Disease (GOLD) strategy ${ }^{1}$ and American Thoracic Society/European Respiratory Society guidelines $^{2}$ recommend inhaled bronchodilators, such as a long-acting muscarinic antagonist (LAMA) alone or in combination with a long-acting $\beta_{2}$-agonist (LABA) or inhaled corticosteroid (ICS), as the mainstay for treatment of patients with chronic obstructive pulmonary disease (COPD). Glycopyrrolate, also known as glycopyrronium bromide, is a fast onset (statistically significant bronchodilation within 5 and 15 minutes post-dose) and long-acting (sustained 24hour bronchodilation) muscarinic antagonist (LAMA), developed for maintenance treatment in patients with COPD. ${ }^{3-5}$ Preclinical studies with glycopyrrolate have demonstrated high affinity and slow dissociation from muscarinic receptors which supports the prolonged bronchodilation effect in patients with COPD. ${ }^{6}$ Various global clinical studies have demonstrated that treatment with glycopyrrolate $63 \mu \mathrm{g}$ (equivalent to glycopyrronium 50 $\mathrm{\mu g}$ ) administered once-daily offers substantial benefit to patients with moderate to severe COPD with improved lung function, dyspnea, health status, rescue medication use, with overall good safety profile $e^{4,5,7}$ and is an approved treatment in more than 80 countries including the European Union, Brazil, Japan, Canada, Switzerland and Australia, excluding the United States. In the United States, a separate phase III clinical trial program with glycopyrrolate $15.6 \mu \mathrm{g}$ (equivalent to glycopyrronium $12.5 \mu \mathrm{g}$ ) was developed that included 2 identical pivotal 12-week efficacy and safety studies, Glycopyrrolate $\mathbf{E}$ ffect on syMptoms and lung function study (GEM1) and GEM2 and a long-term safety study, GEM3. The dose selection was based on a dose-ranging study ${ }^{8}$ showing statistically significant and clinically relevant improvements in trough forced expiratory volume in 1 second $\left(\mathrm{FEV}_{1}\right)$ with twice-daily glycopyrrolate $15.6 \mu \mathrm{g}$ treatment and on discussions with the U.S. Food and Drug Administration (FDA). Seebri ${ }^{\circledR}$ Neohaler ${ }^{\text {TM }}$ (glycopyrrolate) $15.6 \mu \mathrm{g}$ is now approved in the United States for the long-term maintenance treatment of airflow obstruction in patients with COPD.

This manuscript presents the results of the 12-week Phase III GEM2 study that assessed the efficacy and safety of glycopyrrolate $15.6 \mu \mathrm{g}$ b.i.d. compared with placebo in COPD patients with moderate-to-severe airflow limitation (GOLD 2 and 3 according to the GOLD 2011 strategy).

\section{Methods}

\section{Study Design and Treatment}

GEM2 was a 12-week multicenter, randomized, double-blind, parallel-group, placebo controlled study (ClinicalTrials.gov registration number: NCT01715298). The study comprised an initial wash out period (duration of 7 to 1 days depending on washout required for prior medications), a run-in period of 2 weeks, followed by a 12 -week randomized treatment period, and a 30-day safety follow-up period. Patients were randomized after the run-in period to receive glycopyrrolate $15.6 \mu \mathrm{g}$ b.i.d. or placebo (both delivered via the single-dose dry-powder inhaler [the Neohaler ${ }^{\mathrm{TM}}$ device; Novartis, Basel, Switzerland]) for the following 12 weeks using an allocation ratio of 1:1 (Figure 1). Additional details of the randomization and blinding procedures are included in the online supplementary material. This study was conducted at 64 centers within the United States. The first patient was enrolled on November 26, 2012 and the last patient visit was

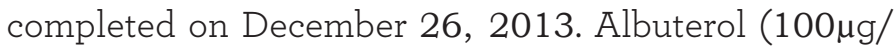
puff administered from the pressurized metereddose inhaler) was allowed as a rescue medication. The continuation of inhaled corticosteroid (ICS) monotherapy at a stable dose regimen was permitted as COPD background therapy. This study was submitted 


\section{Figure 1. Study Design}

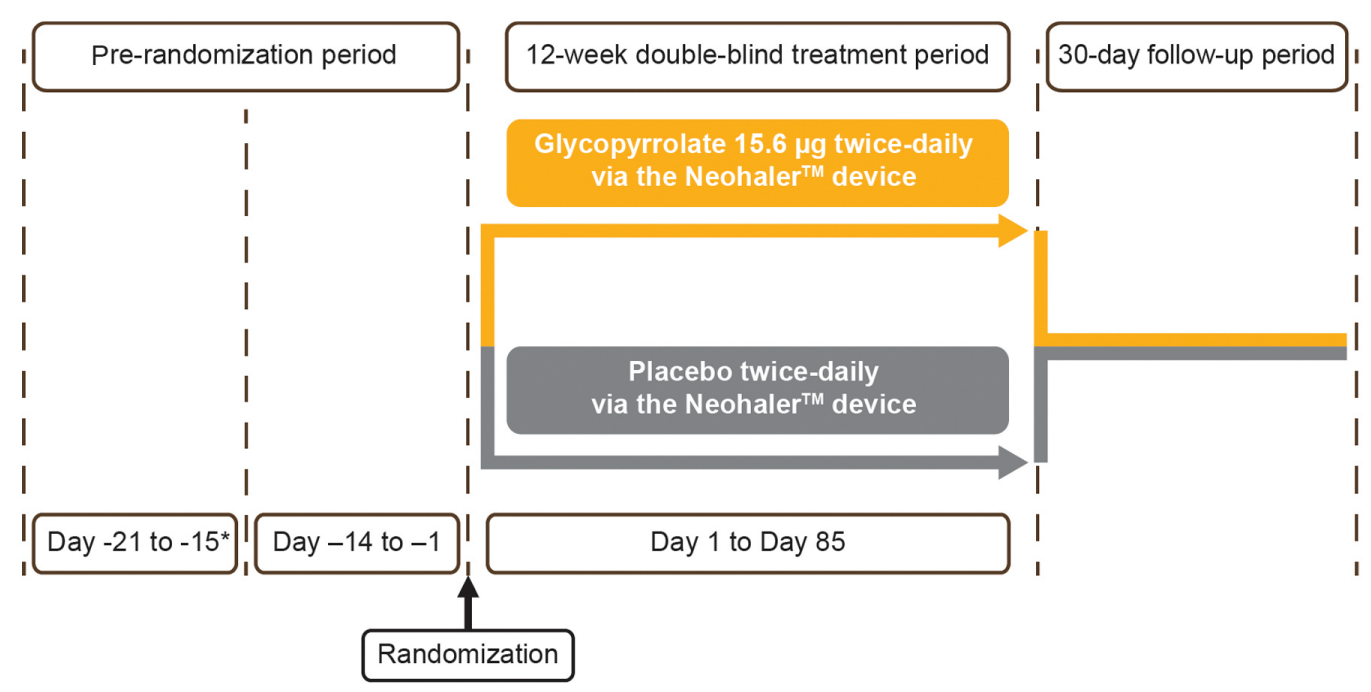

*flexible between 1 to 7 days

to the FDA, was approved by the institutional review boards/independent ethics committees/research ethics boards of participating centers, and was conducted in accordance with the Declaration of Helsinki and the International Conference on Harmonization Good Clinical Practice guidelines. All patients provided written informed consent before participating in the study.

\section{Patients}

The study population included male and female patients aged 40 years or older with moderate-to-severe airflow limitation (GOLD 2 or 3 as defined in GOLD 2011), current or ex-smokers with a smoking history of at least 10 pack years, post-bronchodilator $\mathrm{FEV}_{1} \geq 30 \%$ and $<80 \%$ of predicted value, post bronchodilator $\mathrm{FEV}_{1} /$ forced vital capacity $(\mathrm{FVC}$ ) ratio $<0.70$, and a modified Medical Research Council (mMRC) Dyspnea Scale grade of at least 2 at the run-in visit. Key exclusion criteria included history of asthma and COPD exacerbation requiring treatment with antibiotics, systemic corticosteroids (oral or intravenous), and/ or a hospitalization within 6 weeks before screening or during screening and run-in. Detailed inclusion and exclusion criteria are provided in the online supplementary material.

\section{Study Objectives}

The primary objective was to demonstrate the superiority of glycopyrrolate $15.6 \mu \mathrm{g}$ b.i.d compared with placebo for $\mathrm{FEV}_{1}$ standardized area under curve (AUC) between 0 and 12 hours post dose $\left(\mathrm{FEV}_{1} \mathrm{AUC}_{0-12 \mathrm{~h}}\right)$ at Week 12. The secondary objectives were evaluation of glycopyrrolate compared with placebo in terms of lung function through trough I $\mathrm{FEV}_{1}$ (mean of $\mathrm{FEV}_{1}$ measured l at 23:15h and 23:45h post I previous morning dose) at Day 12 and Week 12, standardized I FEV 1 AUC between 0 and 4 I hours post-dose (AUC $0-4 \mathrm{~h}), 4$ to ' 8 hours post-dose ( $\mathrm{AUC}_{4-8 \mathrm{~h}}$ ), and 8 to 12 hours post-dose $\quad\left(\mathrm{AUC}_{8}\right.$ 12h) on Day 1 and Week 12, $\mathrm{FEV}_{1}$ and $\mathrm{FVC}$ recording at the following time points relative to the morning dose: 5 and $15 \mathrm{~min}, 1,2,4,8$, and 12-hours post dose on Day 1 and Week 12. Additional lung function efficacy objectives were peak FEV 1 and FVC during 4 hours post morning dose on Day 1 and Week 12 and trough FVC on Day 2 and Week 12. The time (min) to achieve $\geq 100 \mathrm{~mL}$ improvement in $\mathrm{FEV}_{1}$ from baseline on Day 1 was also evaluated to determine the onset of action.

Other secondary objectives included evaluation of dyspnea (assessed via the Transition Dyspnea Index [TDI] focal scores), health status (assessed via the St. George's Respiratory Questionnaire [SGRQ] total score) at Week 12, rescue medication use, and COPD symptoms reported using the patient electronic diary (e-diary) over the 12 week treatment period, which also confirmed study compliance. The percentage of patients who achieved the minimal clinically important difference (MCID) in SGRQ total score and TDI focal score in the glycopyrrolate and placebo treated groups was also analyzed. An exploratory objective of the study was to evaluate health status using the COPD assessment test (CAT) at Week 12.

Safety assessments included treatment-emergent adverse events (AEs), monitoring of vital signs (pulse rate, systolic and diastolic blood pressure), electrocardiography, and laboratory analyses (hematology, clinical chemistry assessments, and urinalysis). All serious cardio- and cerebro-vascular events (CCV), atrial fibrillation and atrial flutter, and all cases of death that occurred between randomization 
and the end of the follow-up period were evaluated by an independent adjudication committee. Serious CCV events were adjudicated by major adverse cardiovascular event (MACE) outcome and atrial fibrillation/flutter events were adjudicated based on the new onset or recurrence/persistence of events.

\section{Statistical Analysis}

The full analysis set (FAS) included all randomized patients who received at least 1 dose of the study drug. The per-protocol set (PPS) included all patients in the FAS who did not have any major protocol deviations. The safety set included all patients who received at least 1 dose of the study drug. The FAS was used for the analysis of the primary objective and all other efficacy variables. The PPS was used for the supportive analysis of the primary variable. The safety set was used in the analysis of all safety variables.

$\mathrm{FEV}_{1} \mathrm{AUC}_{0-12 \mathrm{~h}}$ was calculated using the trapezoidal rule divided by length of time (12 hours). ${ }^{9}$ For the analysis of the primary efficacy endpoint (FEV 1 AUC $_{0-12 h}$ ), a mixed model for repeated measures was used. The model contained terms for treatment, baseline $\mathrm{FEV}_{1}$, baseline smoking status, baseline ICS use, visit, treatment $\mathrm{x}$ visit interaction and baseline $\mathrm{FEV}_{1} \mathrm{x}$ visit interaction, with an unstructured covariance matrix. Secondary endpoints were analyzed using the same mixed model as used for the primary endpoint, with the respective baseline values replacing baseline FEV 1 as a covariate as necessary. The proportion of patients who achieved the MCID in the SGRQ total score and TDI focal score was analyzed using a logistic regression model. Details on the sample size estimation are given in the online supplementary material.

\section{Results}

\section{Patient Disposition and Baseline Characteristics}

Of the 1144 patients screened, 432 were randomized to glycopyrrolate $(n=216)$ or placebo $(n=216)$, and 414 patients (96\%) completed the 12-week planned treatment phase (glycopyrrolate, $\mathrm{n}=209$ [96.8\%]; placebo, $n=205$ [94.9\%]). Patients could continue to participate in the 12-week planned treatment phase even if they had permanently discontinued the study medication. The most common reason for study discontinuations was patient decision (glycopyrrolate, $\mathrm{n}=7$; placebo, $\mathrm{n}=9$ ) and 2 patients were discontinued in the placebo group due to lost to follow up.
Patient demographics and baseline clinical characteristics were comparable between the treatment groups (Table 1). Most of the enrolled patients were men $(58.8 \%)$ and white $(86.8 \%)$ with moderate airflow limitation (61.1\%). Most of the patients belonged to the GOLD 2011 Group B (57.6\%) and did not have COPD exacerbations in the year prior to study entry (78.0\%).

\section{Efficacy}

\section{Lung Function}

The study met its primary objective with glycopyrrolate demonstrating significant improvement compared with placebo $(p<0.001)$ in change from baseline in $\mathrm{FEV}_{1}$ AUC $_{0-12 h}$ at Week 12 (Figure 2). Both at Day 1 and Week 12, FEV 1 AUC 0 -12h was higher with glycopyrrolate $(p<0.001)$ compared with placebo, with a statistically significant and clinically meaningful treatment difference $^{10}$ of $119 \mathrm{~mL}$ and $123 \mathrm{~mL}$, respectively (Figure 2). The results of the PPS analysis (Week 12) were consistent with the primary analysis (treatment difference $128 \mathrm{~mL}$; $95 \%$ confidence interval $84,171 \mathrm{~mL}$; $p<0.001)$.

Improvement in $\mathrm{FEV}_{1} \mathrm{AUC}_{0-12 \mathrm{~h}}$ with glycopyrrolate versus placebo in all the subgroups based on age, sex, airflow limitation, baseline smoking status, and ICS use at baseline was generally consistent with the overall study population (Figure 3). Superior bronchodilation with glycopyrrolate was supported by statistically significant improvement in trough $\mathrm{FEV}_{1}$ on Day 2 which was maintained at all timepoints through Week 12 (both Days 85 and 86) (Figure 4) confirming benefits with respect to trough $\mathrm{FEV}_{1}$ for twice-daily glycopyrrolate. Glycopyrrolate showed an early onset of bronchodilation with statistically significant improvements in $\mathrm{FEV}_{1}$ at 5 and 15 minutes post-dose compared with placebo at Day 1 and Week 12 (all $p<0.001$; Figure 5A and 5B). Serial spirometry showed significantly higher improvements in $\mathrm{FEV}_{1}$ with glycopyrrolate versus placebo at all individual post-baseline timepoints from 0 to 12 hours during the study $(p<0.001$ at all timepoints; Figure $5 \mathrm{~A}$ and $5 B$ ). Similarly, FVC at all timepoints from 0 to 12 hours post dose at Day 1 and Week 12 was significantly improved with glycopyrrolate versus placebo (data not shown).

At Week 12, peak FEV 1 and peak FVC for glycopyrrolate were significantly higher compared to placebo (peak FEV 1 treatment difference $148 \mathrm{~mL}$; $p<0.001$ and peak FVC treatment difference 201mL; 


\section{Table 1. Baseline Demographics and Clinical Characteristics}

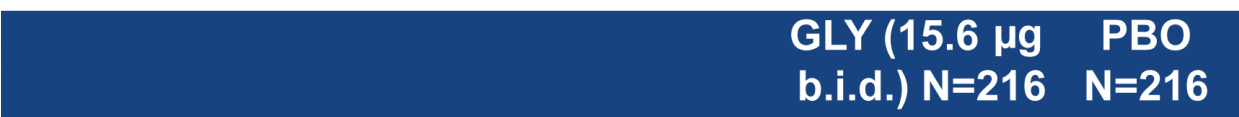

\begin{tabular}{l|rr} 
Age, years & $63.9(8.55)$ & $64.2(8.41)$ \\
\hline Men, n (\%) & $128(59.3)$ & $126(58.3)$ \\
\hline Race, $\mathrm{n}(\%)$ & & \\
\hline White & $190(88)$ & $185(85.6)$ \\
\hline Black & & \\
\hline Smoking History, n (\%) & $101(46.8)$ & $101(46.8)$ \\
\hline Ex-smoker & $115(53.2)$ & $115(53.2)$ \\
\hline Current smoker & & \\
\hline Severity of COPD & \\
\hline GOLD 2 & $139(64.4)$ & $125(57.9)$ \\
\hline GOLD 3 & $73(33.8)$ & $85(39.4)$ \\
\hline
\end{tabular}

Severity of $\mathrm{COPD}^{\mathrm{a}}$, Combined Assessment of COPD, $\mathrm{n}(\%)$

\begin{tabular}{|l|r|r|}
\hline GOLD B & $133(61.6)$ & $116(53.7)$ \\
\hline GOLD D & $78(36.1)$ & $94(43.5)$ \\
\hline uration of COPD (years) & $6.6(4.7)$ & $7.2(5.4)$ \\
\hline
\end{tabular}

COPD Exacerbation History, $\mathrm{n}(\%)$

\begin{tabular}{r|r|r}
\hline 0 & $176(81.5)$ & $161(74.5)$ \\
\hline 1 & $30(13.9)$ & $40(18.5)$ \\
\hline$\geq 2$ & $10(4.6)$ & $15(6.9)$ \\
\hline
\end{tabular}

\begin{tabular}{lll}
\hline Discontinuation of LAMA prior to baseline, $\mathrm{n}(\%)$ & $72(33.3)$ & $54(25.2)$ \\
\hline
\end{tabular}

\begin{tabular}{llll}
\hline Discontinuation of ICS/LABA prior to baseline, $\mathrm{n}(\%)$ & $72(33.3)$ & 74 (34.6) \\
\hline
\end{tabular}

ICS Use at Baseline, $\mathrm{n}(\%)$

$68(31.5) \quad 70(32.4)$

mMRC Dyspnea Scale, n (\%)

Grade 2

Grade 3

Grade 4

$141(65.3)$

66 (30.6)

$8(3.7)$

Number of Pack Years

$50.6(25.34)$

$5.8(1.95)$

BDI Focal Score

SGRQ Total Score

$48.7(16.37)$

CAT Score

$19.1(7.49)$

Pre-bronchodilator $\mathrm{FEV}_{1}$ (L)

$1.31(0.45)$

$1.53(0.49)$

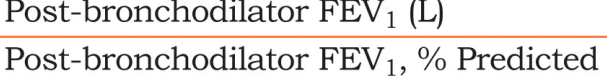

$54.8(12.77)$

Post-bronchodilator $\mathrm{FEV}_{1}$ Reversibility, \% ${ }^{\mathrm{b}}$

$18.3(12.88)$

$130(60.2)$

$75(34.7)$

$10(4.6)$

$50.5(23.59)$

$6(1.93)$

$49.9(16.9)$

$19.3(8.06)$

$1.26(0.46)$

$1.48(0.49)$

$53.8(13.59)$

$21.3(21.27)$

Data are presented as mean (standard deviation) unless otherwise stated.

${ }^{\mathrm{a}}$ COPD severity is based on the GOLD 2011 criteria.

${ }^{\mathrm{b}}$ Assessed after administration of $84 \mu \mathrm{g}$ ipratropium bromide.

$\mathrm{BDI}=$ Baseline Dyspnea Index; $\mathrm{CAT}=\mathrm{COPD}$ assessment test; $\mathrm{COPD}=$ chronic obstructive pulmonary disease; $\mathrm{FEV}_{1}=$ forced expiratory volume in 1 second; $\mathrm{FVC}=$ forced vital capacity; GOLD=Global initiative for chronic Obstructive Lung Disease; GLY=glycopyrrolate;

ICS=inhaled corticosteroid; LABA=long-acting $\beta 2$-agonist; LAMA=long-acting muscarinic antagonist; $\mathrm{mMRC}=$ modified Medical Research Council; $\mathrm{PBO}=$ placebo; SGRQ=St George's Respiratory Questionnaire

Pack years=total years of smoking multiplied by cigarette packs smoked/day $p<0.001 ; \quad$ online supplementary material Table 1).

\section{Health Status and Dyspnea}

With respect to health status, glycopyrrolate showed a statistically significant and clinically relevant improvement in the SGRQ total score compared with placebo $(p<0.001$; Figure 6A) and 29.8\% more patients in the glycopyrrolate group achieved a clinically relevant $\geq 4$ units improvement versus placebo $(p<0.01$; Figure 6B). The TDI focal score showed a numerical reduction in dyspnea with glycopyrrolate compared with placebo (Figure 7A) but the difference was not statistically significant. Overall, more patients receiving glycopyrrolate (46.3\%) achieved the MCID of $\geq 1$ unit in dyspnea than those receiving placebo (39.5\%; Figure 7B). The CAT score significantly improved by -1.5 units at Week 12 (online supplementary material Table 1 ).

\section{Rescue Medication Use and Symptom Scores}

The change from baseline in the use of rescue medication was significantly lower in daily (treatment difference -0.53 puffs/day; $p<0.05)$, daytime (treatment difference -0.30 puffs/day; $p<0.05$ ), and nighttime (treatment difference -0.25 puffs/night; $p<0.05$ ) number of puffs in patients treated with glycopyrrolate compared with placebo over the 12 week treatment period (online supplementary material Table 2). Glycopyrrolate also showed a significant improvement in the daily total symptom score (derived from data recorded in the patient e-diary) compared with placebo (online supplementary material Table 2).

\section{Safety}

Adverse events were reported for 111 (51.4\%) patients in the glycopyrrolate group versus $91(42.5 \%)$ patients in 
the placebo group (Table 2). The incidence of serious AEs (SAEs) was $4.2 \%$ with glycopyrrolate versus $2.3 \%$ with placebo (Table 2). The majority of SAEs were respiratory-related, and the incidence rate was the same across both treatment groups $(1.9 \%$ in both the treatment groups). COPD (exacerbation) was the most

\section{Figure 2. $\mathrm{FEV}_{1} \mathrm{AUC}_{0-12 \mathrm{~h}}$ (FAS)}

(Primary endpoint)

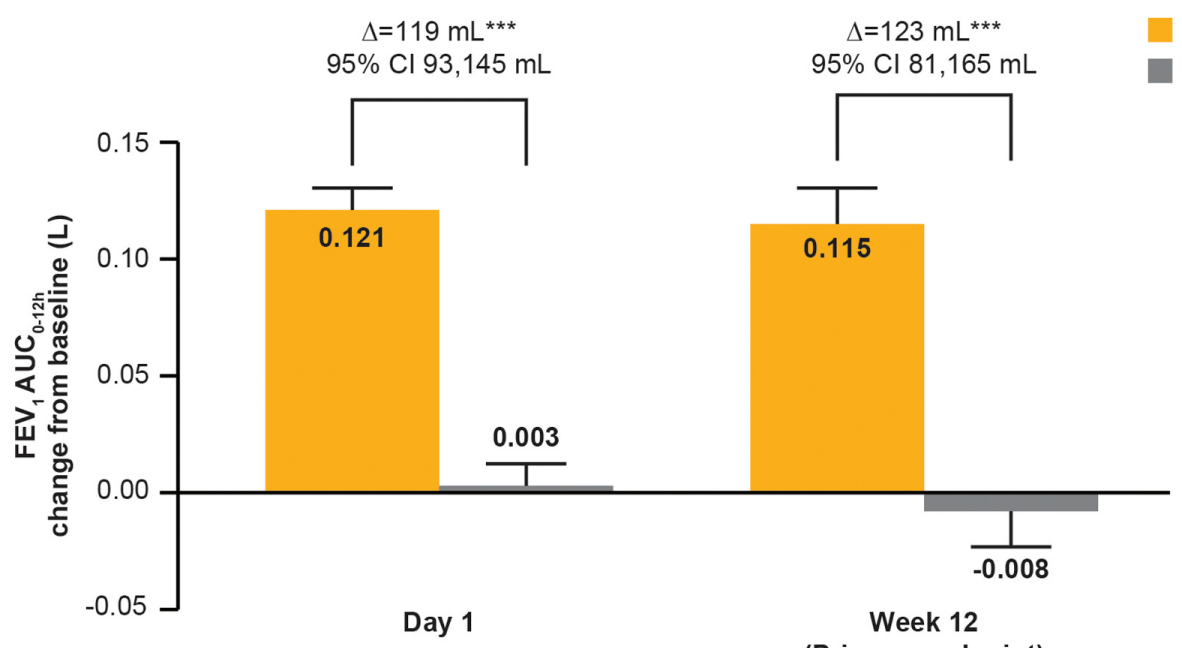

Data are least squares mean (standard error).

$\mathrm{n}=$ number of patients included in the analysis per treatment group.

$\mathrm{AUC}_{0-12 \mathrm{~h}}=$ area under the curve from 0 to 12 hours is defined as the mean $\mathrm{FEV}_{1}$ over 0 to 12 hours;

$\mathrm{FAS}=$ full analysis set; $\mathrm{FEV}_{1}=$ forced expiratory volume in 1 second; GLY=glycopyrrolate;

$\mathrm{PBO}=$ placebo.

*** $p<0.001$ GLY versus PBO

\section{Figure 3. Analysis of The Primary Endpoint FEV $_{1} \mathrm{AUC}_{0-12 \mathrm{~h}}$ Overall and by Subgroup}

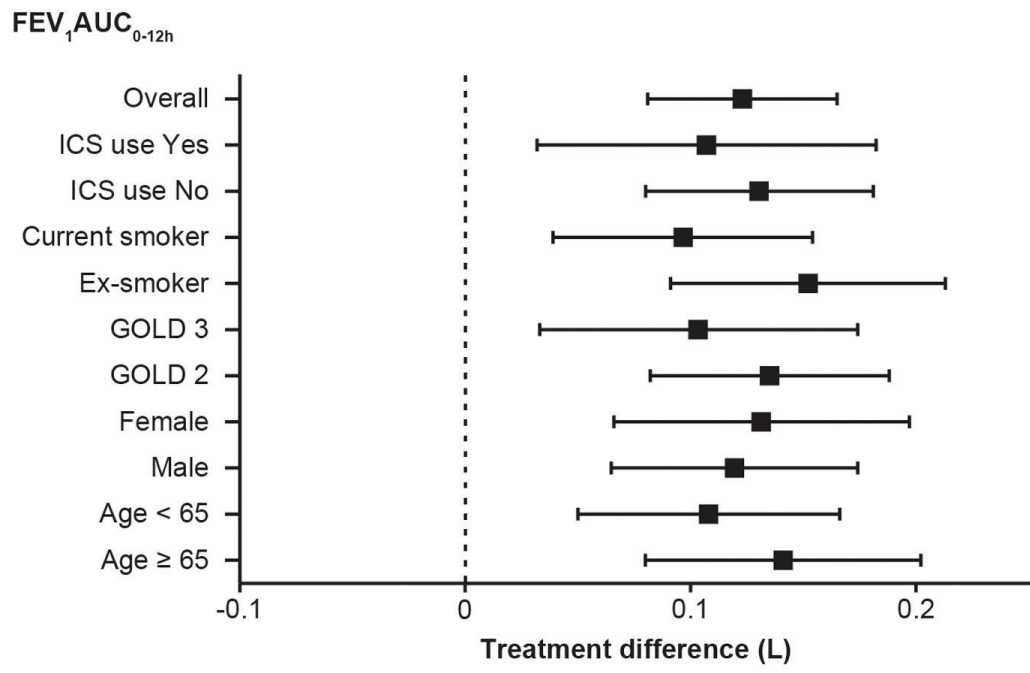

Treatment difference $(95 \% \mathrm{Cl})$

$0.123(0.081,0.165)^{* * *}$ $0.107(0.032,0.182)^{* *}$ $0.130(0.080,0.181)^{\star * *}$ $0.097(0.039,0.154)^{* *}$ $0.152(0.091,0.213)^{* * *}$ $0.103(0.033,0.174)^{* *}$ $0.135(0.082,0.188)^{* * *}$ $0.131(0.066,0.197)^{* * *}$ $0.119(0.065,0.174)^{* * *}$ $0.108(0.050,0.166)^{* * *}$ $0.141(0.080,0.202)^{* * *}$
$\mathrm{CI}=$ confidence interval, GOLD=Global initiative for chronic Obstructive Lung Disease. ICS=Inhaled corticosteroid.

${ }^{* *} p<0.01,{ }^{* * *} p<0.001$ versus placebo commonly reported AE, and was comparable across the treatment groups (glycopyrrolate 20.8\% GLY (n=215) versus placebo 21.5\%; Table BO (n=213) 2). Oropharyngeal related AEs (upper respiratory infection, nasopharyngitis, and oropharyngeal pain) that are commonly seen with orally inhaled LAMAs, occurred at low frequencies in both groups (Table 2). Potential anti-cholinergic AEs occurred infrequently with less than $1.5 \%$ of patients reporting urinary tract infections (Table 2) and less than $1 \%$ reporting dry mouth, constipation and bladder outflow obstruction and urinary retention. The majority of AEs reported during the treatment period were of mild or moderate severity; $5.6 \%$ of patients in the glycopyrrolate group and $2.8 \%$ in the placebo group experienced severe AEs. No death occurred in either group. The proportion of patients who discontinued the study treatment due to AEs (with COPD being the most common $\mathrm{AE}[\geq 1 \%$ in any group] leading to discontinuation) and SAEs was similar between the treatment groups (Table 2). An adjudicated serious CCV AE was reported for 1 patient in the glycopyrrolate group only; this event was adjudicated as MACE and classified as a nonfatal myocardial infarction. No patient in the placebo group had an adjudicated serious CCV event. Atrial fibrillation/flutter events occurred in 4 patients $(1.9 \%)$ in the glycopyrrolate 


\section{Figure 4. Trough FEV 1}

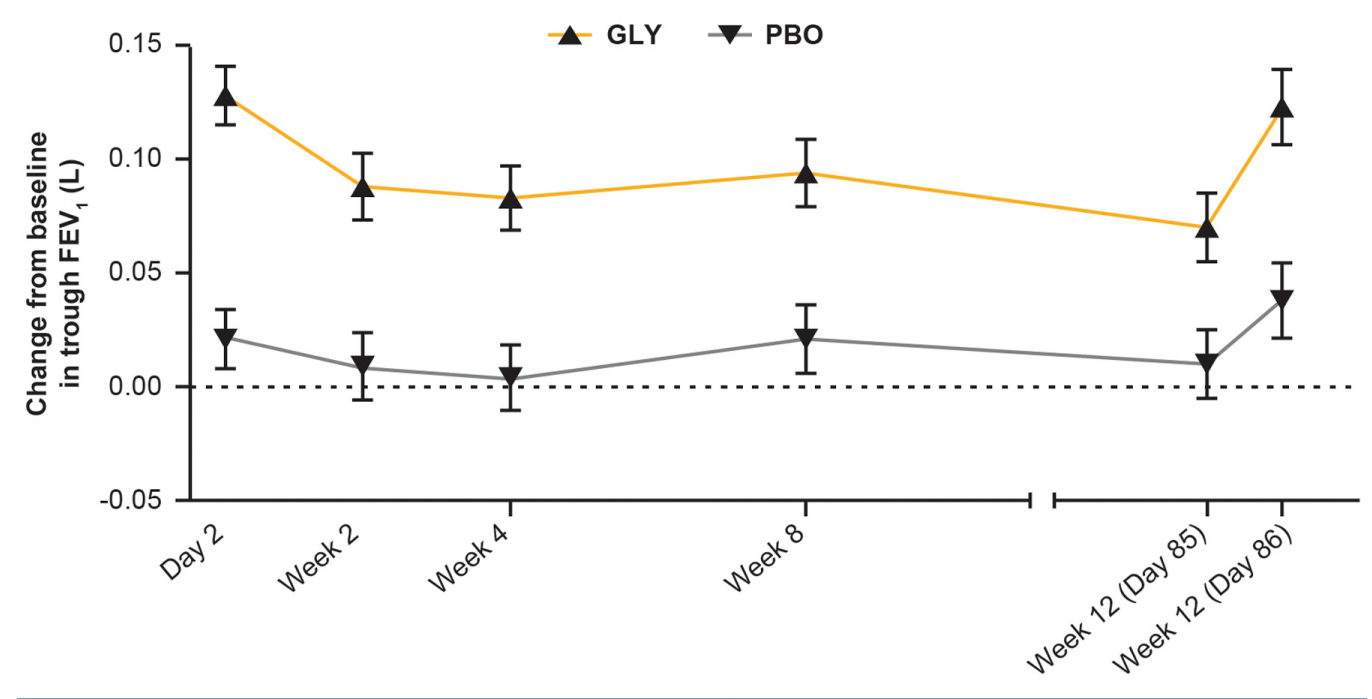

Note break in x-axis. Trough $\mathrm{FEV}_{1}$ was measured at visits on Days 2, 15, 29, 57, 85 and 86.

Differences between GLY and PBO were significant $(\mathrm{p}<0.001)$ at each visit during the treatment period. Trough $\mathrm{FEV}_{1}$ on Day 2 and Day 86 is the mean of $\mathrm{FEV}_{1}$ at 23 hours 15 minutes and 23 hours 45 minutes after the morning dose of the previous day and the mean of $\mathrm{FEV}_{1}$ at -45 minutes and -15 minutes before morning dose at all other visits.

Data are least-squares means; error bars show standard error.

$\mathrm{FEV}_{1}=$ forced expiratory volume in 1 second; GLY=glycopyrrolate; $\mathrm{PBO}=$ placebo; Number of patients included in the analysis, GLY $(n=208-213)$; PBO $(n=204-208)$ efficacious and safe compared with placebo in patients with moderate-to-severe COPD. The selection of the glycopyrrolate dose used in this study was based on the findings of a dose-ranging study in which glycopyrrolate $15.6 \mu \mathrm{g}$ b.i.d. was demonstrated as an effective dose that showed clinically meaningful improvement in trough $\mathrm{FEV}_{1}$ versus placebo. ${ }^{8}$

The study met its primary objective with glycopyrrolate demonstrating superiority to placebo in terms of $\mathrm{FEV}_{1}$ $A C_{0-12 h}$ at Week 12. The magnitude of improvement (glycopyrrolate versus placebo) was $123 \mathrm{~mL}$, which was considered clinically meaningful thus supporting the efficacy of glycopyrrolate $15.6 \mu \mathrm{g}$ b.i.d. Glycopyrrolate was also statistically significantly superior to placebo for this group compared to 1 patient $(0.5 \%)$ in placebo, of which new onset events were reported by 2 patients (0.9\%) in the glycopyrrolate group versus none in the placebo group. The proportion of patients with newly occurring or worsening clinically notable Fridericia's corrected QT interval (QTcF) values was similar between the treatment groups. One patient in the glycopyrrolate group had a QTcF value $>480$ msec. No meaningful differences between treatment groups were seen for laboratory evaluations and clinically notable vital signs.

\section{Discussion}

Bronchodilation with a LAMA and/or LABA is central to the management of COPD. ${ }^{1}$ As per the current clinical guidelines, LAMAs are recommended as a potential first choice therapy, alone or in combination, for patients at high risk of exacerbations. ${ }^{1}$ Glycopyrrolate $15.6 \mu \mathrm{g}$ twice-daily provides an additional LAMA option which has proven to be clinically effective with an early onset of bronchodilatory action and is generally well tolerated with a low incidence of anticholinergic effects, as shown in this study. The overall results of the GEM2 study demonstrate that twice-daily glycopyrrolate is endpoint on Day 1. Other secondary lung function parameters supported the primary endpoint with significant improvements in trough $\mathrm{FEV}_{1}$, pre-dose trough $\mathrm{FEV}_{1}$, peak $F E V_{1}$, peak FVC, and FVC $\mathrm{AUC}_{0}$ 12h. Bronchodilation with glycopyrrolate was evident at Day 1 and was maintained over the 12 -week treatment duration. Compared to the GLOW $1^{4}$ and GLOW $2^{5}$ studies, generally, similar improvements in lung function were seen with glycopyrrolate versus placebo in this study at Week 12 (least square mean [LSM] treatment difference [trough $\mathrm{FEV}_{1}$ ] 108 to $97 \mathrm{~mL}$ in GLOW 1 and GLOW2 studies [12-week data], respectively, versus $86 \mathrm{~mL}$ in this study). Notably, glycopyrrolate in this study has shown similar lung function improvement compared to other twice-daily LAMAs such as aclidinium in the ACCORD COPD II trial (change from baseline in trough $\mathrm{FEV}_{1}$ was 51 and $72 \mathrm{~mL}$ versus placebo at doses of $200 \mu \mathrm{g}$ and $400 \mu \mathrm{g}$ respectively). ${ }^{11}$ Of note, patient baseline characteristics, disease history and severity were comparable between the glycopyrrolate and placebo groups.

As per the GOLD guidelines, an improvement in symptoms is an important goal in the disease 
management of patients with COPD. ${ }^{1}$ Clinical guidelines and systematic reviews suggest that the effectiveness of COPD treatments should not be assessed by lung function alone but should also include a variety of other measures, in particular patient-reported outcomes such as dyspnea, health related quality of life and improved exercise endurance. ${ }^{1,12,13}$ Also, there is clear evidence that improvements in mean trough $\mathrm{FEV}_{1}$ is associated with improvements in SGRQ. ${ }^{14}$ In this study, glycopyrrolate showed significant improvements in health status, mean daily total symptom scores and mean daily rescue medication use over 12 weeks' treatment and these were generally consistent with the known efficacy results of glycopyrrolate from

\section{Figure 5. Serial Measurements of $\mathrm{FEV}_{1}$ by Timepoints (0-12h Post Dose) on (A) Day 1 and (B) Week 12}
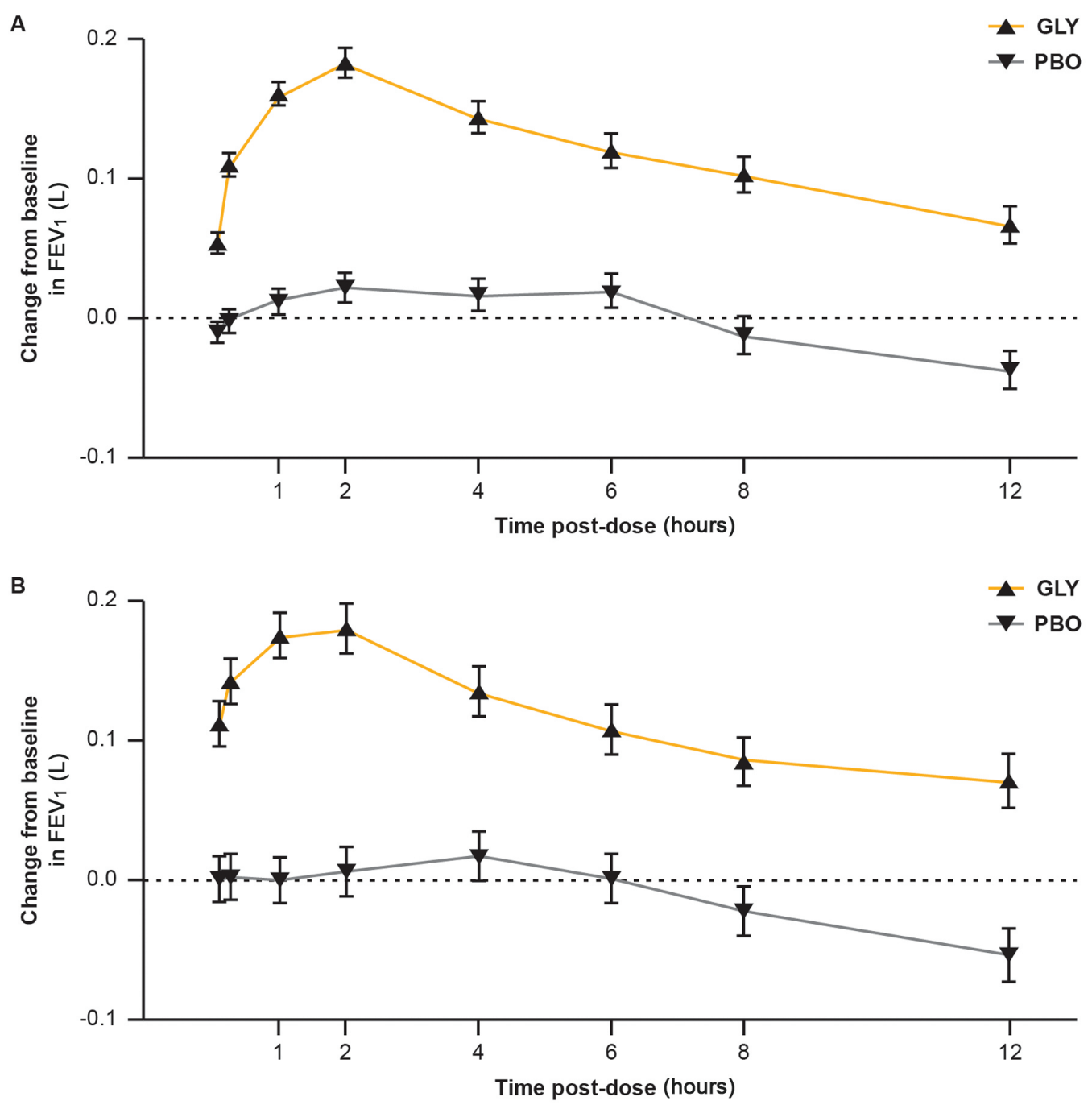

Data are least squares mean (standard error). First 2 time points shown in graph are 5 min and 15 min post dose. Treatment differences: $p<0.001$ for GLY versus PBO at each assessed timepoint.

$\mathrm{FEV}_{1}=$ forced expiratory volume in 1 second; GLY=glycopyrrolate; $\mathrm{PBO}=$ placebo.

(A) Number of patients included in the analysis: GLY $(n=203-215)$. PBO $(n=202-213)$

(B) Number of patients included in the analysis: GLY $(n=203-215)$. PBO $(n=202-213)$ other clinical trials. ${ }^{15}$ The reduced usage of rescue medication in patients receiving glycopyrrolate compared with placebo also suggests an improvement in symptoms. This overall improvement was also reflected in the SGRQ total scores which correlated well with symptom and rescue medication parameters. MCID for the SGRQ is a reduction of 4 units in the total score and is well established. ${ }^{16}$ In this study, the proportion of patients who achieved the MCID in the SGRQ total score was significantly higher with glycopyrrolate than with placebo. In addition, CAT was also used as an exploratory objective to evaluate the improvement in health status and it was observed that glycopyrrolate significantly improved the CAT score compared with placebo. With regards to dyspnea, there was a trend towards improvement in TDI focal score which might have achieved significance with longer study duration.

The safety profile of glycopyrrolate $15.6 \mu \mathrm{g}$ b.i.d observed in this study was consistent with the known safety profile of glycopyrrolate. $^{5,7,8,17,18}$ Antimuscarinic side effects, such as dry mouth, constipation, urinary retention, and urinary tract infections, occurred with a low frequency in both the 
treatment groups. Overall, glycopyrrolate $15.6 \mu \mathrm{g}$ b.i.d. was generally well tolerated over a treatment period of 12 weeks. In spite of some minor imbalances in the frequency of some individual AEs, no clinically relevant differences between the groups could be observed with respect to any type of $\mathrm{AE}$. In patients with moderateto-severe COPD, it had been previously demonstrated that glycopyrrolate was well tolerated and had a similar incidence of MACEs, when compared with placebo or active treatments. ${ }^{4,7}$ Similarly, in this study, based on the adjudicated findings, there was no imbalance in MACEs between the treatment groups and no deaths were observed in either group. The incidence of atrial fibrillation/flutter was low and comparable to that reported with other marketed LAMAs such as umeclidinium and tiotropium. ${ }^{19}$

The study has certain limitations. First, it should be considered that the study duration of 12 weeks

\section{Figure 6. SGRQ Total Score at Week 12 (FAS)}

A

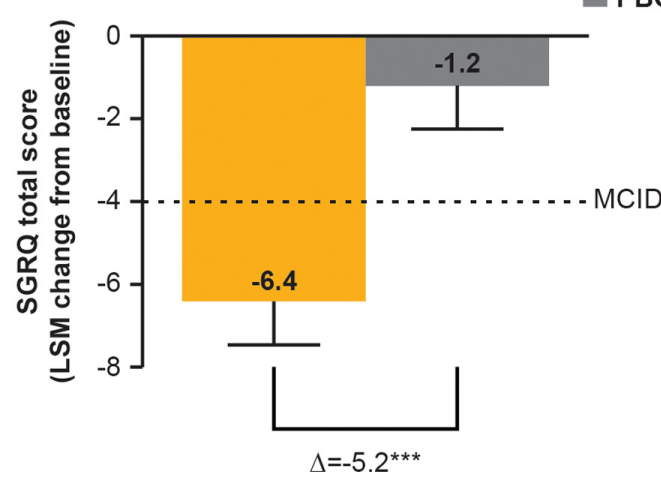

$95 \% \mathrm{Cl}-7.7,-2.7$

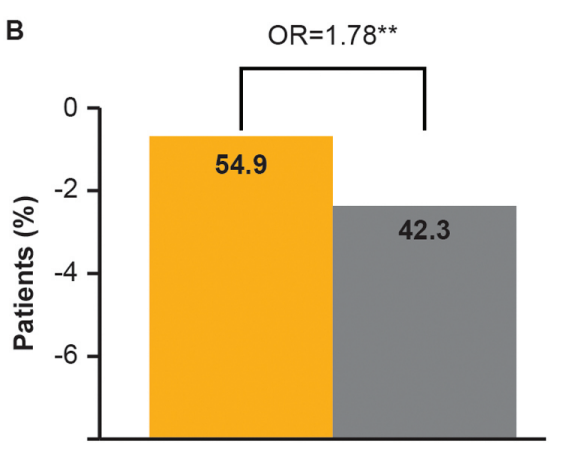
was not powered for analysis of exacerbations or for the assessment of some of the patient-reported outcomes. Similarly, the long-term safety profile could not be established in a 12-week study. Furthermore, no active comparator arm was included. Longer duration studies or studies enriched for COPD exacerbations would be needed to further clarify effects on exacerbations and long term safety findings.

\section{Conclusion}

(B) Percentages of Patients Achieving the Minimum Clinically Important Difference ( $\geq 4$ Units) in SGRQ Score

Data are LSM (standard error) in Figure 6A. FAS=full analysis set; GLY=glycopyrrolate; LSM=least squares mean; $\mathrm{PBO}=$ placebo; SGRQ=St.George's Respiratory Questionnaire; MCID=minimal clinically important difference. OR=odds ratio; ${ }^{* *} p<0.01,{ }^{* * *} p<0.001$, GLY versus $\mathrm{PBO}$

\section{Figure 7. TDI Focal Score at Week 12 (FAS)}
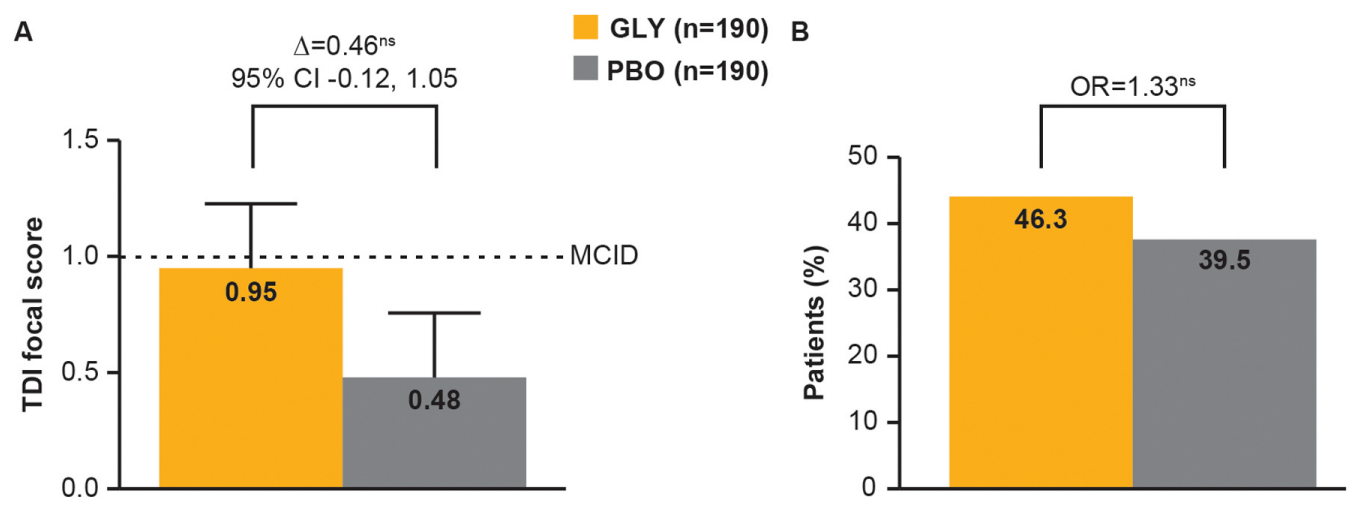

A) TDI Focal Score and

(B) Percentages of Patients Achieving the Minimum Clinically Important Difference ( $\geq 1$ Units) (FAS)

Data are LSM (Standard error) in Figure 7A. FAS=full analysis set; GLY=glycopyrrolate; LSM, least squares mean; ns=not significant; $\mathrm{OR}=$ odds ratio; $\mathrm{PBO}=$ placebo; TDI=transition dyspnea index
The results from the GEM2 study demonstrated that twicedaily glycopyrrolate $15.6 \mu \mathrm{g}$ showed an early onset of action, apparent within 5 and 15 minutes post-dose and sustained 24-hour bronchodilation over 12 weeks compared with placebo. Glycopyrrolate also showed statistically significant improvements in COPD symptoms, health status, and rescue medication use. Overall, glycopyrrolate $15.6 \mu \mathrm{g}$ b.i.d. was generally well tolerated in patients with moderate-tosevere COPD.

\section{Acknowledgements}

This study was sponsored by Novartis Pharma AG. The authors thank the patients who 


\section{Table 2. AEs and Serious AEs $(\geq 1 \%$ of Patients in Either Treatment Group) and Death (Safety Set)}

\begin{tabular}{|c|c|c|}
\hline & $\begin{array}{l}\text { gLY (15.6 } \mu g \\
\text { b.i.d.) } N=216\end{array}$ & $\begin{array}{c}\text { PBO } \\
\text { N=216 }\end{array}$ \\
\hline Patients with any $\mathrm{AE}$ & $111(51.4)$ & $91(42.5)$ \\
\hline \multicolumn{3}{|l|}{ Preferred term } \\
\hline COPD $^{\mathrm{a}}$ & $45(20.8)$ & $46(21.5)$ \\
\hline Upper Respiratory Tract Infection & $7(3.2)$ & $3(1.4)$ \\
\hline Cough & $6(2.8)$ & $6(2.8)$ \\
\hline Back Pain & $5(2.3)$ & $1(0.5)$ \\
\hline Nasopharyngitis & $5(2.3)$ & $2(0.9)$ \\
\hline Oropharyngeal Pain & $5(2.3)$ & $1(0.5)$ \\
\hline Candida Infection & $3(1.4)$ & 0 \\
\hline Cellulitis & $3(1.4)$ & 0 \\
\hline Dizziness & $3(1.4)$ & $2(0.9)$ \\
\hline Urinary Tract Infection & $3(1.4)$ & $3(1.4)$ \\
\hline Rhinitis Allergic & $3(1.4)$ & 0 \\
\hline Sinusitis & $3(1.4)$ & $2(0.9)$ \\
\hline Gastroenteritis Viral & $2(0.9)$ & $3(1.4)$ \\
\hline Diarrhea & $2(0.9)$ & $3(1.4)$ \\
\hline Dyspnea & $3(1.4)$ & $3(1.4)$ \\
\hline Upper Respiratory Tract Infection, viral & $2(0.9)$ & $3(1.4)$ \\
\hline Headache & $2(0.9)$ & $7(3.3)$ \\
\hline Peripheral Edema & $1(0.5)$ & $3(1.4)$ \\
\hline Bronchitis & 0 & $4(1.9)$ \\
\hline Rhinorrhea & $1(0.5)$ & $3(1.4)$ \\
\hline Nasal Congestion & 0 & $5(2.3)$ \\
\hline Patients with SAE(s) & $9(4.2)$ & $5(2.3)$ \\
\hline $\mathrm{COPD}^{\mathrm{a}}$ & $3(1.4)$ & $3(1.4)$ \\
\hline \multicolumn{3}{|l|}{ Discontinuation of Study Treatment } \\
\hline Due to AEs & $10(4.6)$ & $9(4.2)$ \\
\hline Due to SAEs & $2(0.9)$ & $2(0.9)$ \\
\hline Due to non-SAEs & $8(3.7)$ & $7(3.3)$ \\
\hline Deaths & 0 & 0 \\
\hline
\end{tabular}

Data are presented as $\mathrm{n}(\%)$ unless otherwise stated;

$A E s=a d v e r s e$ events; b.i.d. $=$ twice-daily; $\mathrm{COPD}=$ chronic obstructive pulmonary disease; GLY=glycopyrrolate; $\mathrm{PBO}=$ placebo; $\mathrm{SAEs}=$ serious adverse events;

${ }^{a}$ worsening of COPD which includes COPD exacerbation participated, and the staff at the participating clinical centers. The authors also thank Novartis clinical team members Fernando Mota, Pamalar Lewis, Cathy Surdouski, Colleen Spotts, Michael Kleinhoffer and Janet Jenkins for their contributions in executing the study and Vivek Khanna (professional medical writer; Novartis) for assistance in the preparation of this paper.

\section{Declaration of Interest}

EK has served on speaker panels, advisory boards and as consultant for AstraZeneca (Pearl), Amphastar, Forest Laboratories, Sunovion, Boehringer Ingelheim, Teva Labs, Novartis, Mylan and Theravance. TMS has received research funding from Novartis, GlaxoSmithKline, Boehringer Ingelheim, Forest Research Institute, Astra Zeneca, Sunovion, and Pearl Therapeutics. PK has received research support for studies of COPD from Astra Zeneca, Amphastar, Boehringer Ingelheim, Forrest Laboratory, Novartis, Mylan, Sunovian and Teva. AW has no conflict of interests. JHE, FP and PD are employees of the study sponsor, Novartis. $\mathrm{MH}$ is contracted to work for Novartis. All authors participated in the development and writing of the manuscript and take full responsibility for the content of the article. All authors approved the final draft that was submitted. $\mathrm{PD}, \mathrm{JHE}, \mathrm{MH}$ and FP contributed to the design of the study. EK, the principal investigator of the study, has read and commented on the full study report, and had final responsibility for the decision to submit for publication. TMS, PK and AW as investigators of the study, contributed to the writing of each draft of the manuscript. All the investigators were involved in primary data collection. $\mathrm{PD}, \mathrm{JHE}, \mathrm{MH}$ and $\mathrm{FP}$, as employees of the sponsor, contributed to the design and preparation, conduct, analysis and interpretation of the study for the manuscript, and also contributed to the writing of each draft of the manuscript. 


\section{References}

1. Global initiative for chronic Lung Disease (GOLD). Global strategy for the diagnosis, management, and prevention of chronic obstructive pulmonary disease, 2015. GOLD website. www.goldcopd.com. Published 2015. Accessed January 2016.

2. American Thoracic Society and European Respiratory Society. Standards for the Diagnosis and Management of Patients with COPD, 2004. American Thoracic Society website. http:// www.thoracic.org/copd-guidelines/ Published 2004. Accessed January 2016.

3. European Medicines Agency. Seebri breezhaler, glycopyrronium bromide: summary of product characteristics. European Medicines Agency website. http://www.ema.europa.eu/docs/ en_GB/document_library/EPAR_-_Product_Information/ human/002430/WC500133769.pdf. Accessed January 2016.

4. D'Urzo A, Ferguson GT, van Noord JA, et al. Efficacy and safety of once-daily NVA237 in patients with moderate-to-severe COPD: the GLOW1 trial. Respir Res. 2011;12:156. doi: http://dx.doi.org/10.1186/1465-9921-12-156

5. Kerwin E, Hebert J, Gallagher N, et al. Efficacy and safety of NVA237 versus placebo and tiotropium in patients with COPD: the GLOW2 study. Eur Respir J. 2012; 40(5):1106-1114. doi:http://dx.doi.org/10.1183/09031936.00040712

6. Haddad EB, Patel H, Keeling JE, et al. Pharmacological characterization of the muscarinic receptor antagonist, glycopyrrolate, in human and guinea-pig airways. Br J Pharmacol. 1999;127(2):413-20. doi:http://dx.doi.org/10.1038/sj.bjp.0702573

7. D'Urzo A, Kerwin E, Overend T, et al. Once daily glycopyrronium for the treatment of COPD: pooled analysis of the GLOW1 and GLOW2 studies. Curr Med Res Opin. 2014;30(3):493-508. doi:http://dx.doi.org/10.1185/03007995.2013.858618

8. Arievich H, Overend T, Renard D, et al. A novel modelbased approach for dose determination of glycopyrronium bromide in COPD. BMC Pulm Med. 2012;12:74. doi: http://dx.doi.org/10.1186/1471-2466-12-74

9. Mysovskikh IP. Trapezium formula. Encyclopedia of Mathematics website. http://www.encyclopediaofmath.org/ index.php?title=Trapezium_formula\&oldid=12696. Updated February 7, 2011. Accessed January 2016.

10. Cazzola M, MacNee W, Martinez FJ, et al. Outcomes for COPD pharmacological trials: from lung function to biomarkers. Eur Respir J. 2008;31(2):416-469.

doi: http://dx.doi.org/10.1183/09031936.00099306

11. Rennard SI, Scanlon PD, Ferguson GT, et al. ACCORD COPD II: a randomized clinical trial to evaluate the 12-week efficacy and safety of twice-daily aclidinium bromide in chronic obstructive pulmonary disease patients. Clin Drug Invest. 2013;33(12):893904. doi: http://dx.doi.org/10.1007/s40261-013-0138-1

12. Westwood M, Bourbeau J, Jones PW, et al. Relationship between $\mathrm{FEV}_{1}$ change and patient-reported outcomes in randomised trials of inhaled bronchodilators for stable COPD: a systematic review. Respir Res. 2011;12:40.

doi:http://dx.doi.org/10.1186/1465-9921-12-40
13. Glaab T, Vogelmeier C, Buhl R. Outcome measures in chronic obstructive pulmonary disease (COPD): strengths and limitations. Respir Res. 2010;11:79. doi: http://dx.doi.org/10.1186/1465-9921-11-79

14. Global initiative for chronic Lung Disease (GOLD). Global strategy for the diagnosis, management, and prevention of chronic obstructive pulmonary disease, 2014. GOLD website. www.goldcopd.com. Published 2014. Accessed January 2016.

15. Ulrik CS. Once-daily glycopyrronium bromide, a long-acting muscarinic antagonist, for chronic obstructive pulmonary disease: a systematic review of clinical benefit. Int J Obstruct Pulmon Dis. 2012;7:673-678. doi: http://dx.doi.org/10.2147/COPD.S35990

16. Jones PW, Beeh KM, Chapman KR, et al. Minimal clinically important differences in pharmacological trials. Am J Respir Crit Care Med. 2014;189(3):250-255. doi: http://dx.doi.org/10.1164/rccm.201310-1863PP

17. Vogelmeier C, Verkindre C, Cheung D, et al. Safety and tolerability of NVA237, a once-daily long-acting muscarinic antagonist, in COPD patients. Pulm Pharmacol Ther. 2010; 23(5):438-444. doi: http://dx.doi.org/10.1016/j.pupt.2010.04.005

18. Verkindre C, Fukuchi Y, Flemale A, et al. Sustained 24-h efficacy of NVA237, a once-daily long-acting muscarinic antagonist, in COPD patients. Respir Med. 2010; 104(10):1482-1489. doi: http://dx.doi.org/10.1016/j.rmed.2010.04.006

19. Manickam R, Asija A, Aronow WS. Umeclidinium for treating COPD: an evaluation of pharmacologic properties, safety and clinical use. Expert Opin Drug Saf. 2014;13(11):1555-1561. doi: http://dx.doi.org/10.1517/14740338.2014.968550 